\title{
Associations between clinical symptoms, plasma norepinephrine and deregulated immune gene networks in subgroups of adolescent with Chronic Fatigue Syndrome
}

Chinh Bkrong Nguyen ${ }^{1,2}$, Surendra Kumar ${ }^{3}$, Manuela Zucknick ${ }^{4}$, Vessela N. Kristensen ${ }^{1,3,5}$, Johannes Gjerstad $^{6}$, Hilde Nilsen ${ }^{1,5}$, Vegard Bruun Wyller ${ }^{1,2}$

${ }^{1}$ Institute of Clinical Medicine, University of Oslo, 0372 Oslo, Norway

${ }^{2}$ Department of Pediatrics and Adolescent Health, Akershus University Hospital, 1478 Lørenskog, Norway

${ }^{3}$ Department of Genetics, Institute of Cancer Research, Oslo University Hospital Radiumhospitalet, 0310 Oslo, Norway

${ }^{4}$ Oslo Centre for Biostatistics and Epidemiology, Department of Biostatistics, Institute of Basic Medical Sciences, University of Oslo, 0317 Oslo, Norway

${ }^{5}$ Department of Clinical Molecular Biology, Akershus University Hospital, 1478 Lørenskog, Norway

${ }^{6}$ National Institute of Occupational Health, P.O. Box 8149 Dep. N-0033 Oslo, Norway

Corresponding Author:

Chinh Bkrong Nguyen

004746257875

nttchinhb@gmail.com 


\begin{abstract}
Background: Chronic fatigue syndrome (CFS) is one of the most important causes of disability among adolescents while limited knowledge exists on genetic determinants underlying disease pathophysiology.
\end{abstract}

Methods: We analyzed deregulated immune-gene modules using Pathifier software on whole blood gene expression data (29 CFS patients, 18 controls). Deconvolution of immune cell subtypes based on gene expression profile was performed using CIBERSORT. Supervised consensus clustering on pathway deregulation score (PDS) was used to define CFS subgroups. Associations between PDS and immune, neuroendocrine/autonomic and clinical markers were examined. The impact of plasma norepinephrine level on clinical markers over time was assessed in a larger cohort (91 patients).

Results: A group of 29 immune-gene sets was shown to differ patients from controls and detect subgroups within CFS. Group 1P (high PDS, low norepinephrine, low naïve CD4+ composition) had strong association with levels of serum C-reactive protein and Transforming Growth Factor-beta. Group 2P (low PDS, high norepinephrine, high naïve CD4+ composition) had strong associations with neuroendocrine/autonomic markers. The corresponding plasma norepinephrine level delineated 91 patients into two subgroups with significant differences in fatigue score.

Conclusion: We identified 29 immune-gene sets linked to plasma norepinephrine level that could delineate CFS subgroups. Plasma norepinephrine stratification revealed that lower levels of norepinephrine were associated with higher fatigue. Our data suggests potential involvement of neuroimmune dysregulation and genetic stratification in CFS. 
KEYWORDS: Chronic Fatigue Syndrome, Myalgic Encephalomyelitis, immune alteration, Pathifier, pathway deregulation analysis, neurotransmitter, norepinephrine, CD4+ naïve $\mathrm{T}$ cells, heart rate variability, neuro-immune dysregulation, fatigue score, genetic stratification. 


\section{Introduction}

The Chronic Fatigue Syndrome (CFS) is characterized by disproportional fatigue after exertions, musculoskeletal pain, cognitive impairment and other symptoms. The prevalence of CFS is expected to be between $0,006 \%-3 \%{ }^{1}$ and the disease affects more females than males. CFS is a major threat towards adolescent health, with strong negative impact on academic development and family life ${ }^{2}$.

The aetiology of CFS is unknown; however, several studies have linked its pathophysiology to perturbation in the immune system including altered function of B cells, Tregs, neutrophils and NK cells ${ }^{3,4}$ and altered levels of certain cytokines associated with disease duration ${ }^{5}$. Recently, we identified subtle downregulation of genes encoding proteins that function in adaptive immune responses and upregulation of genes belonging to innate responses in whole blood mRNA of young CFS patients compared with healthy controls ${ }^{6}$. Additionally, CFS is characterized by neuroendocrine alterations including enhanced sympathetic nervous activity, increased plasma levels of catecholamines and attenuation of the hypothalamus-pituitary-adrenal axis (HPA-axis) ${ }^{7-9}$

Increasing evidences in systems biology have suggested that studying higher degree of molecular interactions might shed light on the underlying mechanisms of complex diseases ${ }^{10,11}$. Approaches studying groups of genes that are functionally related could help to identify co-expression modules or elucidate perturbed biological networks. Such analyses incorporate quantitative profiles of each molecule into higher order of their interactive map such as gene sets, protein networks, or signaling pathways. In turn, associations between deregulated modules/networks and clinical symptoms could be the effective tools to generate hypotheses concerning disease mechanism. Pathway-based genomic models have been demonstrated to give better indicators of prognosis for a number of diseases ${ }^{12,13}$. From available gene expression data, pathway-based analysis such as Pathifier can identify deregulated pathways or disrupted gene network using measurement of pathway deregulated scoring (PDS). 
Generally, there is a lack of association studies between clinical and genomic features in CFS. Another important point is the heterogeneity regarding the CFS diagnostic criteria ${ }^{14-16}$. These criteria may be related to different subtypes of the disease ${ }^{17}$. We hypothesized that identification of differential gene expression modules could help to delineate subgroups of CFS.

Therefore, the aims of the present study were two-fold: 1) To identify immune deregulation at the gene set level in adolescent CFS by an exploratory computational approach making use of whole blood gene expression data; and 2) To delineate subgroups in a cohort of adolescent CFS patients by examining clusters of immune gene module expression.

\section{Methods}

\section{Study design}

Adolescent CFS patients ( $\mathrm{n}=120$, mean age 14.96 years, $62 \%$ females) and healthy controls $(\mathrm{n}=68$, mean age 14.65 years, $59 \%$ females) were recruited to the NorCAPITAL-project (The Norwegian Study of Chronic Fatigue Syndrome in Adolescents: Pathophysiology and Intervention Trial; ClinicalTrials ID: NCT01040429), a cross-sectional study of CFS patients and healthy adolescents combined with a randomized controlled trial (RCT) of low-dose clonidine vs. placebo in the CFS group

${ }^{18}$. Clonidine is an alpha-adrenoreceptor agonist which reduces neural sympathetic outflow, thereby lowering heart rate, blood pressures and total peripheral resistance ${ }^{19}$. In NorCAPITAL, CFS patients allocated to the active treatment arm of the RCT received oral clonidine capsules $25 \mu \mathrm{g}$ or $50 \mu \mathrm{g}$ twice daily, respectively, for body weight below/above $35 \mathrm{~kg}$. CFS patients were summoned for investigations at three time-points: Baseline (prior to treatment allocation in the RCT), at 8 weeks (during treatment), and at 30 weeks (after treatment discontinuation). Healthy controls were seen only once. Ethical approval of the study was granted by the Regional Committee for Ethics in Medical Research, the Norwegian Data Inspectorate, the Norwegian Directorate of Health, and the Norwegian Medicines Agency. 


\section{Recruitment}

Details of the recruitment procedure and inclusion/exclusion criteria are described elsewhere ${ }^{18}$. Briefly, all hospital pediatric departments in Norway $(n=20)$, as well as primary care pediatricians and general practitioners, were invited to refer CFS patients aged 12-18 years consecutively to our study center. A standard form required the referral unit to confirm the result of clinical investigations considered compulsory to diagnose pediatric CFS according to national Norwegian recommendations (pediatric specialist assessment, comprehensive hematology and biochemistry analyses, chest x-ray, abdominal ultrasound, and brain MRI). Also, the referring units were required to confirm that the patient a) was unable to follow normal school routines due to fatigue; b) was not permanently bedridden; c) did not have any concurrent medical or psychiatric disorder that might explain the fatigue; d) did not experience any concurrent demanding life event (such as parents' divorce) that might explain the fatigue; e) did not use pharmaceuticals (including hormone contraceptives) regularly. The patients were not screened for childhood trauma experiences. Patients considered eligible to this study were summoned to a clinical encounter at our study center after which a final decision on inclusion was made. In agreement with clinical guidelines, we applied a 'broad' case definition of CFS, requiring three months of unexplained, disabling chronic/relapsing fatigue of new onset ${ }^{20}$. We did not require that patients meet any other accompanying symptom criteria. A group of healthy controls having the same distribution of sex and age as the CFS patients was recruited from local schools.

\section{Immune, neuroendocrine/autonomic, and clinical markers}

Serum level of C-reactive protein, high-sensitive assay (hsCRP) was analyzed by particle-enhanced immunoturbidimetric assay (CRP Latex HS, Roche Diagnostics, Indianapolis, IN, USA). Plasma levels of Transforming Growth Factor $\beta$ (TGF- $\beta$ ) including three isoforms of TGF- $\beta$ (TGF- $\beta 1$, TGF$\beta 2$ and TGF- $\beta 3$ ) was analyzed using Bio-Plex Human TGF- $\beta$ 3-plex (Bio-Rad Laboratories Inc., Hercules, CA, USA) and performed by the Bio-Plex200 system. Neuroendocrine/autonomic markers (plasma and urine levels of norepinephrine and epinephrine) were collected as previously described and measured by the use of high performance liquid chromatography (HPLC) with a reversed-phase 
column and glassy carbon electrochemical detector (Antec, Leyden Deacade II SCC, Zoeterwoude, The Netherlands) ${ }^{6,21}$. The low frequency:high frequency (LF/HR) ratio of supine heart rate variability is an index of sympathetic versus parasympathetic heart rate control; it was calculated from 4 minutes ECG recordings of horizontal-laying participants connected to the Task Force Monitor (Model 3040i, CN Systems Medizintechnik, Graz, Austria) as described elsewhere ${ }^{22}$. The clinical symptom of fatigue (a main complaint among CFS patients) was charted by the Chalder Fatigue Questionnaires (CFQ); a validated instrument consisting of 11 items scored on 0-3 Likert scales ${ }^{18}$. Global functional ability was assessed by the Functional Disability Inventory (FDI), consisting of 15 items scored on 0-4 Likert scales ${ }^{18}$.

\section{Whole blood gene expression data}

Random sampling of 29 CFS patients and 18 healthy controls at time of inclusion (baseline) with similar demographical and clinical parameters compared to the NorCapital cohort were used for whole blood RNA extraction ${ }^{6}$. These two groups were homogenous for age and gender (Table 1).

All RNAs with RIN-value $>7$ were used for gene expression measurements, as described elsewhere ${ }^{6}$. After removing globin and ribosomal RNAs, mRNA sequencing was performed on the Illumina HiSeq 2500 according to the protocol of TruSeq Stranded mRNA single-end sequencing. Normalization of gene expression data from this whole blood was performed using RUVSeq and differential expression analysis was done using DESeq2 as described previously ${ }^{6}$.

\section{Gene sets and pathway database}

Gene sets were downloaded from the Molecular Signatures Database (MSigDB version 5.0) from the C7-immunologic collection consisting of 4872 gene sets. This C7 collection contains list of gene sets that are known to be related to the immune system from 389 published transcriptomic studies of immunologic cell states and immune dysregulation in mouse and human (254 studies in mouse and 135 in human). It was built based on manually annotated gene sets from a wide range of cell states, experimental evidences and genetic alteration that represent cell types, states, and perturbations within 
the immune system ${ }^{22}$. The name of each gene set is context-specific with direction of change for each group of genes per experiment.

\section{Bioinformatics and statistical analysis}

All computational analyses were performed in the R environment of version 3.4 .2 (2017-09-28) and with Graphpad Prism version 6 (GraphPad Software, CA, USA). Two-sided p-values less than 0.05 were considered statistically significant.

\section{Pathway analysis}

Pathway Deregulation Score (PDS) for a given pathway or gene set based on gene expression data was computed by Pathifier Bioconductor package 1.16.0 ${ }^{23}$ implemented in R. The Pathifier method ${ }^{23}$ uses pathway annotation resources such as the Molecular Signatures Database MSigDB ${ }^{24}$ to estimate the PDS of each pathway per sample. MSigDB contains 17786 gene sets spanning across canonical pathways, biological processes and interactive gene sets of the immune system. These gene sets are divided into 8 main collections and several sub-collections based on database types, ontology resources or positional annotation. From the normalized gene expression data, Pathifier transformed per gene quantification into pathway-level measurement and compared their deregulated profile with the corresponding pathway profile of the healthy controls. PDS was ranged from 0 to 1 in which higher values of PDS reveal higher degree of deregulation compared to reference (the healthy controls group).

The normalized RNA-Seq data consisting of 20729 genes from 47 samples (29 patients and 18 controls) was used as input for Pathifier. Output of Pathifier analysis was a matrix of which rows represented the pathways or gene sets and columns were the corresponding PDS of each pathway/gene set per sample.

\section{Visualization of the deregulated gene sets}


Unsupervised clustering on Euclidean distance of PDS values was constructed using Ward's agglomerative linkage method (ward.D2) and visualized as a heatmap by hierarchical clustering using the heatmap 3 package version 1.1.1 ${ }^{25}$. To avoid individual deviation in the cluster, samples that had technical errors such as PDS clustered together in the reverse way compared with all the remaining patients/controls or lacked measurement in several pathways were considered as outliers/ extreme cases (Fig S1).

\section{Deconvolution of immune cell subtypes from gene expression data}

Characterization of the immune cell subtype composition from whole blood gene expression was performed using CIBERSORT software with the inclusion of LM22 background containing 22 immune cell types and 1000 permutation for statistical estimation ${ }^{26}$.

\section{Consensus clustering of the deregulated gene sets}

Classification of the top deregulated gene sets of 43 samples (26 patients, 17 controls after removing the extreme cases) into clusters was identified using ConsensusClusterPlus package version 1.40.0 ${ }^{27}$. Consensus clustering of the deregulated gene sets from the analysis on C7 MSigDB was performed by three different clustering algorithms (Hierarchical clustering, K-means, Partition around medoids (PAM)) with $80 \%$ item resampling, $80 \%$ gene resampling, 100 replications and an evaluation of two to six clusters using Euclidean distance. Dissimilarities between each object in each cluster were inspected by measuring the Silhouette value using the Cluster package version $2.0 .6^{28}$.

\section{Establishment of the three gene set clusters and their centroids}

The best clustering algorithm chosen was K-means $(\mathrm{K}=3)$ following Silhouette values and output of ConsensusClusterPlus. Results for the hierarchical clustering and PAM are not shown, but these results can be reproduced with the code provided in https://github.com/chinhbn/pathifier. The three centroids were the centers of three clusters computed by K-means clustering $(K=3)$ above. Summary of the best 
consensus clustering result was performed by an unsupervised clustering employing ward.D method and Euclidean distance of the centroids using pheatmap package version 1.0.8 ${ }^{29}$.

\section{Partitioning of patients}

Partitioning of patients was done using the PDS values of the centroids (matrix of three centroids and 26 patients) by employing K-means consensus clustering on their Euclidean distance with $80 \%$ item and $80 \%$ gene set resampling, 100 replicates and 2-4 K-means clusters evaluation.

Identification of the intersectional genes from differential gene expression analysis and differential pathway analysis

Genes that were found to be differentially expressed in our previous study ${ }^{6}$ and belong to the deregulated gene sets found by Pathifier were matched and identified. The normalized expression variances were analyzed by using the first two principal components from Principal component analysis (PCA).

\section{Gene Ontology enrichment analysis}

The 60 genes that belong to those deregulated gene sets were visualized as nodes in their associated network using Cytoscape software version 3.5.1 and the top Gene Ontology (GO) encoding for biological process (BP) terms were enriched using ClueGO version $2.5^{30}$. Enrichment for significant BP GO terms with all evidence codes was performed by a two-sided hypergeometric test employing the mid-p-value method. Adjustment for multiple testing was done by controlling the false discovery rate following Benjamini-Hochberg method. Min GO level and Max GO level were set as default with no GO Fusion and Kappa Score Threshold was set as 0.3.

\section{Statistical testing}


Differences across groups regarding background, immune, neuroendocrine/autonomic and clinical markers were performed by t-test statistics or Mann-Whitney $U$ test as appropriate.

Deregulated pathway measurement data were presented as PDS Mean, Standard deviations (SD) and 95\% Confidence Interval (95\% CI). PDS mean of each gene set across three different groups were compared using Kruskal-Wallis test. Pairwise post-hoc comparisons were performed by nonparametric Dunn's tests using Benjamini-Hochberg stepwise adjustment for the multiple pairwise comparisons.

\section{Associations of the deregulated gene sets with other markers}

Spearman correlation matrices were applied to explore the associations between immune marker (hsCRP), TGF- $\beta$, neuroendocrine/autonomic markers (plasma norepinephrine, LF/HF ratio), and clinical markers (CFQ score, FDI score) with each of the 29 gene sets, using the cor function in R.

\section{Associations of patient subgroups stratified by plasma norepinephrine level with clinical markers over time}

In the entire NorCAPITAL cohort, clinical markers were compared between two subgroups of patients that differ by the baseline plasma norepinephrine levels. A cutoff defined by the first quartile of plasma norepinephrine of Group 2P (1754,75 pmol/L) delineated the NorCapital cohort $(\mathrm{n}=120)$ into two subgroups: Low norepinephrine $(\mathrm{n}=59)$, High norepinephrine $(\mathrm{n}=61)$. After removing participants lacking at least two time-points of the clinical measurements, two subgroups homogenous for age and gender ([Low norepinephrine group, $n=43]$, [High norepinephrine $=48]$ ) were included.

The CFQ and FDI scores over time (baseline, week 8 and week 30) were compared between norepinephrine subgroups by repeated measures ANOVA (RM ANOVA) in SPSS. The model included plasma norepinephrine group, gender as the main between-subject effects and age, treatment allocation (clonidine/placebo) as covariates. Interaction effects of treatment allocation with outcome 
were included in the RM ANOVA model. The effect of genotype differences was not investigated as the genetic frequencies of the assessed adrenergic receptor and catechol $O$-methyl transferase polymorphisms were not significantly different between subgroups (Table S1).

\section{Results}

The computational workflow from visualization of Pathifier result and clustering of deregulated C7 gene sets, patients partitioning as well as GO enrichments for differential genes is described as in Fig S1.

Primary unsupervised clustering of PDS values suggested removal of extreme cases including three patients and one control samples (Fig S2). The Pathifier result below therefore includes 26 patients and 17 healthy individuals.

\section{Patient characteristics}

CFS patients had similar distribution of sex, age and body mass index (BMI) as well as immune markers $(14.96 \pm 1.32$ years old, $19.99 \pm 3.30 \mathrm{BMI})$ compared to healthy controls ( $14.65 \pm 1.46$ years old, 19.41 1.94 BMI) (Table 1). Regarding neuroendocrine/autonomic measurements, plasma norepinephrine, plasma epinephrine, and urine norepinephrine/creatinine were significantly higher in patients compared to controls ( $\mathrm{p}<0.05$, Table 1$)$. Also, clinical markers (CFQ total score and median FDI) were significantly different in patients compared to healthy controls $(\mathrm{p}<0.001$, Table 1).

\section{Pathway deregulation quantification and visualization}

In our analysis, we specifically aligned our normalized gene expression data with gene sets of the $\mathrm{C} 7$ immunological collection of MSigDB.

Hierarchical clustering cooperating 4309 measurable $\mathrm{C} 7$ gene sets showed relatively good separation between a subgroup of patients and controls (Fig 1A, Fig S2). In addition, this initial inspection of the data suggested that a panel of 29 deregulated gene sets had potential to differ patients from controls as 
well as to subtype patients (Fig 1A). Hereafter, our pathway deregulation analysis only focused on these top 29 deregulated immune-gene sets.

\section{Hierarchical clustering and consensus clustering of the C7 PDS data}

By Pathifier analysis, the 29 deregulated gene sets primarily delineate patients into two subgroups: a subgroup of 12 patients (hereafter labeled "Group 1P") and a second group comprising the remaining 14 patients (“Group 2P”) (Fig 1A). Since these 29 gene sets may contain overlapping genes, consensus clustering was applied in order to obtain robust classification of gene sets (Fig S3A, S3C). Silhouette measures on the potential classification confirmed that the grouping of the 29 gene sets into three Kmeans clusters resulted in the best grouping (Fig S3B). The centroids of these K-means consensus clusters were subsequently used in the patient-partitioning step (Fig S4, Table S2).

Additionally, consensus clustering on the gene sets revealed Group 1P had higher immunological deregulation at the gene expression level than Group 2P (Fig 2A, Fig 2C, Table 2). CFS patients in Group 2P and the healthy controls appeared to be more heterogeneous (Fig 1A, Table 2).

\section{Pathway deregulated score (PDS) of the 29 gene sets across groups}

The PDS per gene set across groups are displayed in Table 2. Among the 29 gene sets, the PDS values of 28 gene sets were found to be significantly different between the two potential patient subgroups (1P vs. 2P) and between Group 1P compared to healthy controls.

\section{Patient partitioning based on the K-means clustering}

Consensus clustering using the $3 \mathrm{~K}$-means centroids classified the 26 patients into three subgroups (Fig S4 and Table S3). However, as the third grouping contained only 2 samples which appeared to lack PDS values for several pathways, these two patients were not considered in the 1P vs. $2 \mathrm{P}$ comparison, which therefore consisted of 12 patients per group (Fig 2A-E). 


\section{Differentially expressed genes component of the 29 gene sets}

A total of 176 genes have previously been reported as differentially expressed in whole blood from CFS patients (FDR 10\%, fold change $0.82-1.25)^{6}$. Among these genes, a total of 60 (50 up-regulated and 10 down-regulated genes) belong to one or more of the identified 29 deregulated immune-gene sets (Table S4). The first principal component of the PCA projecting the variances of the normalized expression counts explained $54.69 \%$ of the total variability of the 60 genes compared between the two patient subgroups (Fig 2B).

Gene set enrichment by ClueGO and CluePedia revealed that the immunological signatures were dominated by innate immunity (negative regulation of viral genome replication, positive regulation of interleukin-1 beta production) (Fig 1B, Table S5). Negative regulation of viral genome replication was the top immune GO (G0:0045071) and was enriched consistently in our repeated analysis with the presentation of FAM111A (encoding for Family with sequence similarity 111 member A), IFI16 (encoding for Interferon gamma inducible protein 16), PLSCR1 (Phospholipid scramblase 1). Another identified immune process was lymph node development (GO:0048535).

The associations between immune markers, neuroendocrine/autonomic markers, clinical markers and the 29 gene sets

Plasma norepinephrine levels $(\mathrm{pmol} / \mathrm{L})$ differed across subgroups of patients (Group 1P: Mean 1520 [95\% CI 1262-1778], Group 2P: Mean 2392 [95\% CI 1797-2736]) as well as between all CFS patients compared to healthy controls (Patients: Mean 2090 [95\% CI 1735-2445], Healthy controls: Mean 1540 [95\% CI 1352-1729], $p$-value $=0.039$, Table 1). While their immunological deregulation measured by PDS was higher (Fig 2A, Table S2), Group 1P had significant lower plasma norepinephrine level (Fig. 2D, p-value $=0.0463)$, and significant lower fraction of CD4+ naïve T cells (Fig. 2E, $p$-value $=0.014)$ compared to Group 2P. The plasma norepinephrine level of group 1P $(\mathrm{n}=12)$ was equal to the healthy control group ( $\mathrm{n}=17)$ (data not shown). 
In Group 1P, there was negative correlations between several deregulated gene sets and hsCRP (such as $\quad \mathrm{r}=\quad-0.592 \quad$ between GSE21927_SPLENIC_VS_TUMOR_MONOCYTES_FROM_C26GM_TUMOROUS_MICE_BAL BC_DN and hsCRP) as well as plasma TGF- $\beta 3$ ( $\mathrm{r}=-0.664$ between GSE21927_SPLENIC_VS_TUMOR_MONOCYTES_FROM_C26GM_TUMOROUS_MICE_BAL BC_DN and TGF- $\beta 3$ ) (Table S6, S7). Intriguingly, a number of gene sets in Group 1P was positively associated with fatigue score (Table S8). In addition, 15 out of 29 gene sets were negatively correlated with neutrophil cell counts $(|\mathrm{r}|>0.5)$ (data not shown).

In Group 2P, several gene sets were positively associated with plasma norepinephrine and the LF/HF ratio (Table S8); these associations were not found in the Group 1P. Furthermore, there were strong associations between several gene sets and functional disability score; again, these associations appeared to be rather specific for Group 2P (Table S9). In addition, within Group 2P, the association between the 29 immune-gene sets, the blood cell counts and immune markers were much weaker than in Group 1P (except for the CD4 naïve T cells).

\section{Immune cell composition based on gene expression data}

The fraction of immune cell subtypes in each sample was predicted by CIBERSORT as shown in Table S10. The fraction of naïve CD4+ T cells was significantly different between the two subgroups $1 \mathrm{P}$ vs $2 \mathrm{P}$ ( $p$-val=0.014, Mann Whitney U test) $($ Fig $2 E)$ but invariant when all patients $(n=26)$ were compared to all controls $(n=17)$ or between each subgroup vs controls. The fractions of remaining immune cell subtypes, including B-cell subtypes were similar between the two patient subgroups.

The associations of plasma norepinephrine subgroups with clinical markers over time 
The CFQ total score was found to be significantly different between the two norepinephrine groups ([Low norepinephrine group, $\mathrm{n}=43]$, [High norepinephrine =48]) over time, as shown in Fig 3A. A similar, non-significant trend was observed for the FDI score over time (Fig 3B).

\section{Discussion}

In this study we identified a group of 29 immune-gene sets which differs a subset of CFS patients $(n=12)$ from controls and which comprises 60 out of 176 genes that were previously found to be differentially expressed in CFS patients ${ }^{6}$. This genetic cluster (referred to as "immune-gene network") revealed two subgroups within CFS patients (Group 1P: n=12, Group 2P: n=12), which in turn differed significantly in plasma norepinephrine level and naïve CD4+ composition. The corresponding plasma norepinephrine level was able to classify a larger patient cohort $(n=91)$ into two subgroups with significant differences in fatigue questionnaires score.

One differentially regulated immune-gene set is the GSE6269_HEALTHY_VS_FLU_INF_PBMC_UP, which contains CREBZF and CXCR5. CREBZF encodes CREB/ATP BZIP, a transcription factor with AP1-like component, which is involved in MAPK-ERK and B cell receptor signaling pathways ${ }^{31}{ }^{32}$. The protein product of CXCR5, CXC motif chemokine receptor 5, is known to be expressed in mature B cells for guiding B cell migration into secondary lymph nodes. Another interesting gene set is the GSE21927_SPLENIC_VS_TUMOR_MONOCYTES_FROM_C26GM_TUMOROUS_MICE_BAL BC_DN which contains 9 up-regulated genes (RSRP1, CLK4, TMEM260, ARHGAP15, WSB 1, FBXL5, ZNF586, CLK1, NKTR). This gene set is consistently correlated with a number of immune markers as well as neuroendocrine/autonomic markers. The protein product of $A R H G A P 15$ is a Rho GTPase activator and phospholipid binding protein of which its over-expression was found to be involved in actin stress fibers and cell contraction ${ }^{33}$ that might be relevant to the fatigue symptom ${ }^{34}$. 
Interesting, through GO enrichment, innate and adaptive immunity genes was revealed to be connected through the bridge of TLR4 and TNF- $\alpha$ (Figure 1B, Table S5), which supports our previous findings on the co-presence of up-regulated innate immune responses and down-regulated B-cell differentiation and survival ${ }^{6}$. Computational deconvolution of immune cell subtypes indicated that the latter was not related to a reduced representation of B-cell subsets in the sample material. RNA surveillance against viruses represented the top biological processes (GO: 0045071), which includes FAM111A, IFI16, PLSCR1. Of note, PLSCR1 was validated by qPCR to be upregulated in the patient group compared to the control group ${ }^{6}$. This gene encodes Phospholipid scramblase 1 (PLSCR1), an interferoninducible protein, which was suggested to mediate antiviral activity against DNA and RNA viruses through the activation of type 1 Interferon response ${ }^{35}$.

The present study also suggested subgrouping in CFS and a role of the immune-autonomic interaction in CFS. Patients in Group 1P were found to have significantly higher PDS values and lower naïve CD4+ subset than patients in Group 2P, suggesting stronger deregulation of the immune-gene networks. Furthermore, Group 2P had significantly higher level of plasma norepinephrine compared to patients in Group 1P, whose plasma norepinephrine levels were comparable to healthy controls. Our result might indicate that enhanced sympathetic nervous activity, causing elevated norepinephrine levels, may play a role in maintaining immune homeostasis in CFS individuals in the Group P2, whereas the lower level of norepinephrine in Group 1P corresponds with a more extensive alteration of immune pathways in this group. Thus, this finding is in line with previous observations and hypotheses of the immune-brain communication ${ }^{36-38}$ and also agrees with general notions of norepinephrine as a mediator of anti-inflammatory effects ${ }^{37}$. In previous studies, norepinephrine was elevated in adolescent CFS as compared to healthy controls ${ }^{18,39}$ and was suggested to potentially have a central role in neuroendocrine-immunological crosstalk in CFS pathogenesis. Interestingly, a very recent study in mice has revealed the unique molecular machinery of the sympathetic neuronassociated macrophages (SAMs) which participate in controlling regional norepinephrine levels ${ }^{40}$. SAMs express the $\beta$-adrenergic receptors and sodium-dependent neurotransmitter transporter (NAT1/solute carrier protein SLC6A2) for reuptake of norepinephrine, thereby terminate its effect 
and contribute in maintaining extracellular norepinephrine balance. In addition, SAMs were suggested to play a key role in the prevention of over-secretion of norepinephrine into the blood stream during enhanced sympathetic activity ${ }^{40}$. Interestingly, mutation of the SLC6A2 impairing synaptic norepinephrine clearance was suggested to be associated with orthostatic intolerance ${ }^{41}$ which is a common clinical phenomenon in CFS ${ }^{42}$.

Strikingly, these two patient subgroups differed in their patterns of associations between the immunegene sets and other biomarkers. Several strong associations between markers of autonomic nervous activity (plasma norepinephrine, ratio of heart rate frequencies $(\mathrm{LF} / \mathrm{HF})$ ) and the immune-gene sets were found almost exclusively in Group P2, whereas immune markers (hsCRP) were associated to several immune-gene sets in Group 1P. Also, in Group 1P only, six gene sets were found to be significantly associated with TGF- $\beta 3$ (Table S7). This is consistent with our previous finding on the association between expression levels of the CXC motif chemokine receptor 5 gene (CXCR5), B cellactivating factor receptor gene $(B A F F-R)$ and TGF $-\beta^{21}$. Of note, TGF- $\beta$ plays an important role in the regulation of cell proliferation, differentiation, apoptosis and is related to a number of human diseases. The LF/HF ratio (an index of sympathetic vs. parasympathetic heart rate control) was positively correlated with 8 gene sets in Group 2P, but only 2 gene sets in Group 1P (Table S8). This corroborates our previous finding of an association between gene expression and the LF/HF index among CFS patients (Nguyen et al, 2017), and is also consistent with other reports of an inverse relationship between the inflammatory markers and reduced parasympathetic nervous activity ${ }^{43}$. Taken together, these results support previous findings of a potential associated deregulation in endocrine/autonomic and immune system in CFS ${ }^{9,44,45}$. Thus, the molecular mechanisms underlying such alteration should be scrutinized in further studies.

Finally, difference in plasma norepinephrine levels between Group 1P ( $\mathrm{n}=12)$ vs. Group 2P $(n=12)$ appeared to be useful in classifying a larger patient group from the same project into two subgroups (Low vs. High norepinephrine) which differed significantly in the fatigue score over time (Fig 3A); a 
non-significant trend was observed for functional disability (Fig 3B). Generally, the cohort with the highest plasma norepinephrine levels (similar to Group 2P) had less serious symptoms and disabilities, supporting the idea of increased sympathetic activity in CFS patients as a compensatory mechanism in order to maintain immune homeostasis. At week 8, patients who received clonidine treatment could result in the lower level of plasma norepinephrine ${ }^{18}$ compared to baseline. In our preliminary analysis using the RM ANOVA model of CFQ/FDI over time, the effect of treatment allocation between the low and high norepinephrine groups was not significant, thus both clonidine and placebo groups were pooled together in the final RM ANOVA analysis as shown in Fig 3.

With respect to genetic determinants that are associated with CFS, very few studies put genes into their interactive contexts, except for one study in which integrated networks and genetic variants analysis identified a network of 20 candidate genes associated with the Tryptophan hydroxylase 2 gene (TPH2) ${ }^{46}$, which encodes the THP2 protein that catalyzes the rate limiting step in the serotonin biosynthesis pathway. By integrating weighted gene co-expression network analysis on microarray data and genetic variants of 76 CFS patients, the study identified the gene network to be associated with CFS severity ${ }^{46}$. While most of the efforts in molecular CFS studies have been looking at single associated loci, the fluctuation coming from networks of genes or proteins may be more relevant. Therefore, in addition to per gene measurement, the present study shows that it is worthwhile to calculate the effect of the corresponding deregulated hubs of genes. Importantly, the delineation of CFS patients into two such subgroups with differences in plasma norepinephrine and clinical symptoms suggests that stratification of participants might be useful to reduce the heterogeneity in CFS.

In conclusion, we identified a 29 immune-gene sets linked to plasma norepinephrine and naïve CD4+ T cells which can delineate subgroups of CFS patients and the potential involvement of neuro-immune dysregulation in CFS pathophysiology. The delineation of patients into two subgroups with differential norepinephrine levels suggests plasma norepinephrine might be used to stratify patient in future clinical studies; e.g. novel therapeutic strategies might be designed and tested in patients with similar levels of norepinephrine. 


\section{Acknowledgements}

We thank Tom Eirik Mollnes and Judith Anita Ludviksen at the Research Laboratory, Nordland Hospital, Bodø, and Faculty of Health Sciences, K.G. Jebsen Thrombosis Research and Expertise Center, University of Tromsø, Norway for the TGF- $\beta 3$ analyses; Lene Alsøe at Akershus University Hospital for comments on the manuscript; Maria Johansson at Institute of Neuroscience and Physiology, University of Gothenburg, Sweden for helpful discussion. 


\section{References}

1. Cleare AJ, Reid S, Chalder T, Hotopf M, Wessely S. Chronic fatigue syndrome. BMJ Clin Evid. 2015;2015.

2. Kennedy G, Underwood C, Belch JJ. Physical and functional impact of chronic fatigue syndrome/myalgic encephalomyelitis in childhood. Pediatrics. 2010;125(6):e1324-1330.

3. Bradley AS, Ford B, Bansal AS. Altered functional B cell subset populations in patients with chronic fatigue syndrome compared to healthy controls. Clin Exp Immunol. 2013;172(1):73-80.

4. Brenu EW, Huth TK, Hardcastle SL, et al. Role of adaptive and innate immune cells in chronic fatigue syndrome/myalgic encephalomyelitis. Int Immunol. 2014;26(4):233-242.

5. Montoya JG, Holmes TH, Anderson JN, et al. Cytokine signature associated with disease severity in chronic fatigue syndrome patients. Proc Natl Acad Sci U S A. 2017;114(34):E7150-E7158.

6. Nguyen CB, Alsoe L, Lindvall JM, et al. Whole blood gene expression in adolescent chronic fatigue syndrome: an exploratory cross-sectional study suggesting altered B cell differentiation and survival. J Transl Med. 2017;15(1):102.

7. Jason L, Sorenson M, Sebally K, et al. Increased HDAC in association with decreased plasma cortisol in older adults with chronic fatigue syndrome. Brain Behav Immun. 2011;25(8):1544-1547.

8. Papadopoulos AS, Cleare AJ. Hypothalamic-pituitary-adrenal axis dysfunction in chronic fatigue syndrome. Nat Rev Endocrinol. 2011;8(1):22-32.

9. Wyller VB, Vitelli V, Sulheim D, et al. Altered neuroendocrine control and association to clinical symptoms in adolescent chronic fatigue syndrome: a crosssectional study. J Transl Med. 2016;14(1):121.

10. Barabasi AL, Gulbahce N, Loscalzo J. Network medicine: a network-based approach to human disease. Nat Rev Genet. 2011;12(1):56-68.

11. Vidal M, Cusick ME, Barabasi AL. Interactome networks and human disease. Cell. 2011;144(6):986-998.

12. Huang $\mathrm{S}$, Yee $\mathrm{C}$, Ching T, Yu H, Garmire LX. A novel model to combine clinical and pathway-based transcriptomic information for the prognosis prediction of breast cancer. PLoS Comput Biol. 2014;10(9):e1003851.

13. Sun Z, Wang L, Eckloff BW, et al. Conserved recurrent gene mutations correlate with pathway deregulation and clinical outcomes of lung adenocarcinoma in never-smokers. BMC Med Genomics. 2014;7:32.

14. Asprusten TT, Fagermoen E, Sulheim D, et al. Study findings challenge the content validity of the Canadian Consensus Criteria for adolescent chronic fatigue syndrome. Acta Paediatr. 2015;104(5):498-503.

15. Jason LA, McManimen S, Sunnquist M, Brown A, Newton JL, Strand EB. Examining the Institute of Medicine's Recommendations Regarding Chronic Fatigue Syndrome: Clinical Versus Research Criteria. J Neurol Psychol. 2015;2015(Suppl 2).

16. Rowe PC, Underhill RA, Friedman KJ, et al. Myalgic Encephalomyelitis/Chronic Fatigue Syndrome Diagnosis and Management in Young People: A Primer. Front Pediatr. 2017;5:121. 
17. Jason LA, Corradi K, Torres-Harding S, Taylor RR, King C. Chronic fatigue syndrome: the need for subtypes. Neuropsychol Rev. 2005;15(1):29-58.

18. Sulheim D, Fagermoen E, Winger A, et al. Disease Mechanisms and Clonidine Treatment in Adolescent Chronic Fatigue Syndrome: A Combined Cross-sectional and Randomized Clinical Trial. JAMA Pediatr. 2014;168(4):351-360.

19. Yasaei R, Saadabadi A. Clonidine. StatPearls. Treasure Island (FL)2018.

20. Health RCoPaC. Evidence based guidelines for the management of CFS/ME (chronic fatigue syndrome/myalgic encephalopathy) in children and young adults. . London: Royal College of Paediatrics and Child Health. 2004.

21. Wyller VB, Nguyen CB, Ludviksen JA, Mollnes TE. Transforming growth factor beta (TGF-beta) in adolescent chronic fatigue syndrome. J Transl Med. 2017;15(1):245.

22. Godec J, Tan Y, Liberzon A, et al. Compendium of Immune Signatures Identifies Conserved and Species-Specific Biology in Response to Inflammation. Immunity. 2016;44(1):194-206.

23. Drier Y, Sheffer M, Domany E. Pathway-based personalized analysis of cancer. Proc Natl Acad Sci U S A. 2013;110(16):6388-6393.

24. Liberzon A, Subramanian A, Pinchback R, Thorvaldsdottir H, Tamayo P, Mesirov JP. Molecular signatures database (MSigDB) 3.0. Bioinformatics. 2011;27(12):1739-1740.

25. Zhao S, Guo Y, Sheng Q, Shyr Y. Advanced heat map and clustering analysis using heatmap3. Biomed Res Int. 2014;2014:986048.

26. Newman AM, Liu CL, Green MR, et al. Robust enumeration of cell subsets from tissue expression profiles. Nat Methods. 2015;12(5):453-457.

27. Wilkerson MD, Hayes DN. ConsensusClusterPlus: a class discovery tool with confidence assessments and item tracking. Bioinformatics. 2010;26(12):15721573.

28. Maechler M, Rousseeuw, P., Struyf, A., Hubert, M., Hornik, K. cluster: Cluster Analysis Basics and Extensions. R package version 2.0.6. 2017.

29. Kolde R. Pheatmap: pretty heatmaps. R package 2012.

30. Bindea G, Mlecnik B, Hackl H, et al. ClueGO: a Cytoscape plug-in to decipher functionally grouped gene ontology and pathway annotation networks. Bioinformatics. 2009;25(8):1091-1093.

31. Bodnarchuk TW, Napper S, Rapin N, Misra V. Mechanism for the induction of cell death in ONS-76 medulloblastoma cells by Zhangfei/CREB-ZF. J Neurooncol. 2012;109(3):485-501.

32. GeneCards. CREB/ATF BZIP Transcription Factor. Weizmann Institute of Science.

33. Seoh ML, Ng CH, Yong J, Lim L, Leung T. ArhGAP15, a novel human RacGAP protein with GTPase binding property. FEBS Lett. 2003;539(1-3):131-137.

34. MacIntosh BR, Holash RJ, Renaud JM. Skeletal muscle fatigue--regulation of excitation-contraction coupling to avoid metabolic catastrophe. J Cell Sci. 2012;125(Pt 9):2105-2114.

35. Dong B, Zhou Q, Zhao J, et al. Phospholipid scramblase 1 potentiates the antiviral activity of interferon. J Virol. 2004;78(17):8983-8993.

36. Watkins LR, Goehler LE, Relton JK, et al. Blockade of interleukin-1 induced hyperthermia by subdiaphragmatic vagotomy: evidence for vagal mediation of immune-brain communication. Neurosci Lett. 1995;183(1-2):27-31.

37. Sternberg EM. Neural regulation of innate immunity: a coordinated nonspecific host response to pathogens. Nat Rev Immunol. 2006;6(4):318-328. 
38. Andersson U, Tracey KJ. Neural reflexes in inflammation and immunity. J Exp Med. 2012;209(6):1057-1068.

39. Wyller VB, Evang JA, Godang K, Solhjell KK, Bollerslev J. Hormonal alterations in adolescent chronic fatigue syndrome. Acta Paediatr. 2010;99(5):770-773.

40. Pirzgalska RM, Seixas E, Seidman JS, et al. Sympathetic neuron-associated macrophages contribute to obesity by importing and metabolizing norepinephrine. Nat Med. 2017;23(11):1309-1318.

41. Shannon JR, Flattem NL, Jordan J, et al. Orthostatic intolerance and tachycardia associated with norepinephrine-transporter deficiency. $N$ Engl J Med. 2000;342(8):541-549.

42. Medicine Io. Beyond Myalgic Encephalomyelitis/Chronic Fatigue Syndrome: Redefining an Illness. Beyond Myalgic Encephalomyelitis/Chronic Fatigue Syndrome: Redefining an Illness. Washington (DC)2015.

43. Cooper TM, McKinley PS, Seeman TE, Choo TH, Lee S, Sloan RP. Heart Rate Variability Predicts Levels of Inflammatory Markers: Evidence for the Vagal AntiInflammatory Pathway. Brain Behav Immun. 2015;49:94-100.

44. Wyller VB, Eriksen HR, Malterud K. Can sustained arousal explain the Chronic Fatigue Syndrome? Behav Brain Funct. 2009;5:10.

45. Wyller VB, Barbieri R, Saul JP. Blood pressure variability and closed-loop baroreflex assessment in adolescent chronic fatigue syndrome during supine rest and orthostatic stress. Eur J Appl Physiol. 2011;111(3):497-507.

46. Presson AP, Sobel EM, Papp JC, et al. Integrated weighted gene co-expression network analysis with an application to chronic fatigue syndrome. BMC Syst Biol. 2008;2:95. 


\section{Figure legends}

\section{Fig 1. The top 29 deregulated gene sets and the corresponding biological processes}

A. Unsupervised clustering on pathway deregulation score (PDS) of 43 individuals (26 patients and 17 controls) identified clustering of a subset of patients into Group 1P. Horizontal annotation: Patients in red, Healthy controls in green. Increasing plasma norepinephrine levels range from white to dark gray. Left vertical annotation: Selective differentially expressed immune-genes.

B. Significant biological processes enriched from 60 differentially expressed genes (DE genes) (Table S4) from Pathifier and gene expression analysis. Red nodes are genes from the original list. GO groups are corresponding with the enrichment statistics in Table S5.

Fig 2. Differences between two subgroups of CFS patients. Group IP (Low plasma Norepinephrine and High PDS) vs Group $2 P$ (High plasma Norepinephrine and Low PDS). Group 1P in Red, Group 2P in Blue

A. Comparison of Pathway Deregulation Score (mean PDS) of centroid clusters across patient groups, $\mathrm{p}$-val $<0.0001$ by $\mathrm{t}$ test.

B. PCA plot inspecting variance of normalized expression of 60 differentially expressed genes.

C. Unsupervised clustering of the centroids of K-means cluster.

D. Plasma Norepinephrine ( $\mathrm{pmol} / \mathrm{L}$ ) difference between two subgroups of patients. Group 1P: Mean 1520 pmol/L (95\% CI 1262-1778), Group 2P: Mean 2392 pmol/L (95\% CI 1797-2736). p-val $=0.0463$, Mann-Whitney U-test.

E. Naïve CD4 T cells composition differences between Group 1P vs Group 1P. Group 1P: Mean 0.2036 (95\% CI $0.1795-0.2287)$, Group 2P: Mean 0.2509 (95\% CI $0.2044-0.2974)$. p-val = 0.014, Mann-Whitney U-test.

Fig 3. Association between plasma norepinephrine stratification in the NorCAPITAL population and clinical symptoms 
A. Chalder Fatigue Questionnaires scores (CFQ) over time were significantly higher in the Low plasma norepinephrine group $(\mathrm{n}=39)$ compared to the High plasma norepinephrine group $(\mathrm{n}=40)$. Data are given as mean \pm SEM ( $\mathrm{p}$-val $=0.023$, test of between-subjects effect, repeated measures ANOVA).

B. Differences in Functional Disability Inventory score (FDI) over time between the low $(n=43)$ and high $(n=48)$ plasma norepinephrine were shown. Data are given as mean $\pm \operatorname{SEM}(\mathrm{p}$-val $=0.472$, test of between-subjects effect, repeated measures ANOVA). 
Figure 1

A

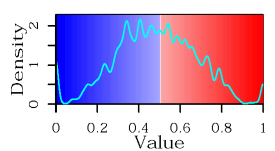

dist=euclidean : hclust_method=ward.D2

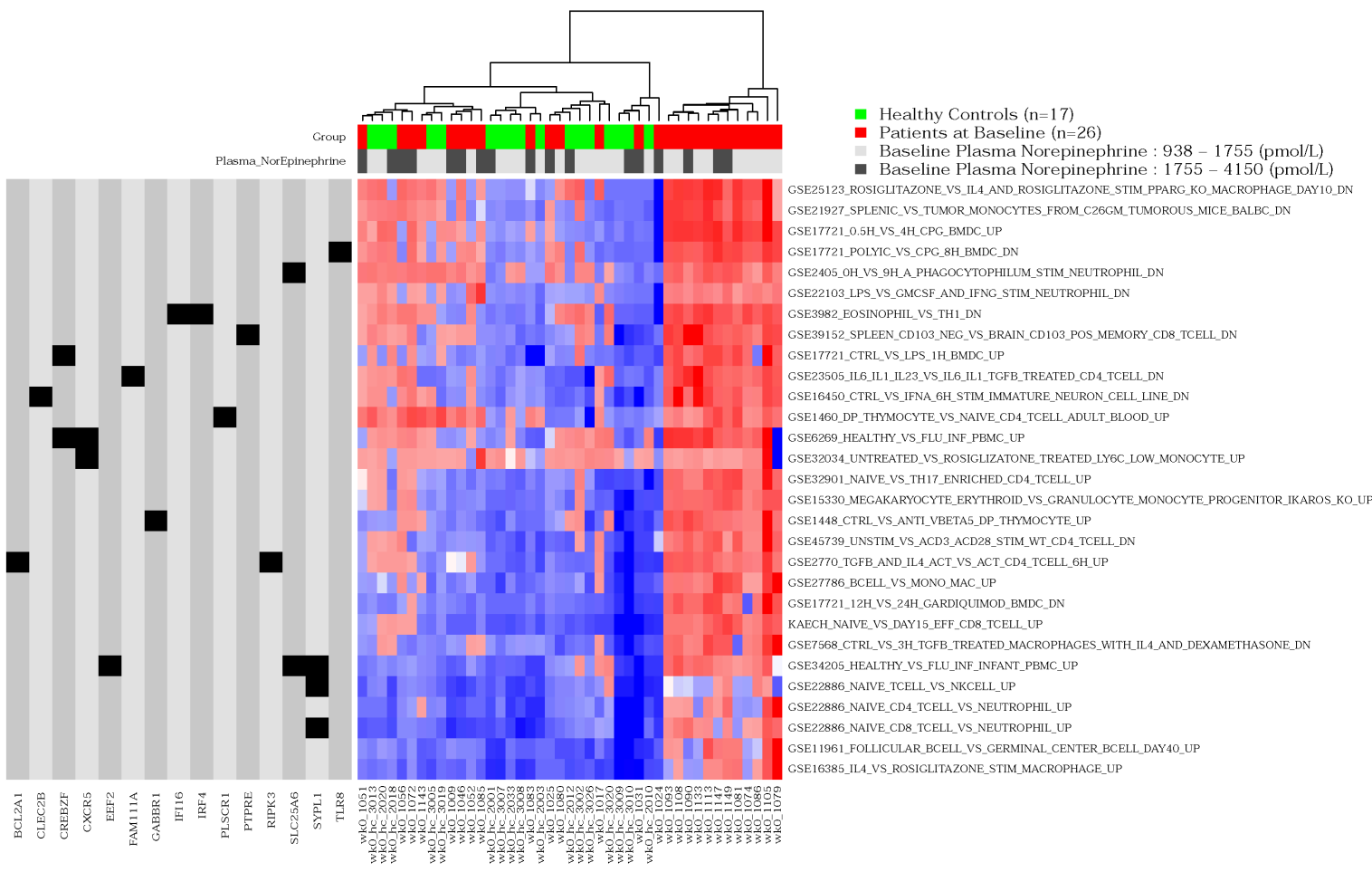

B

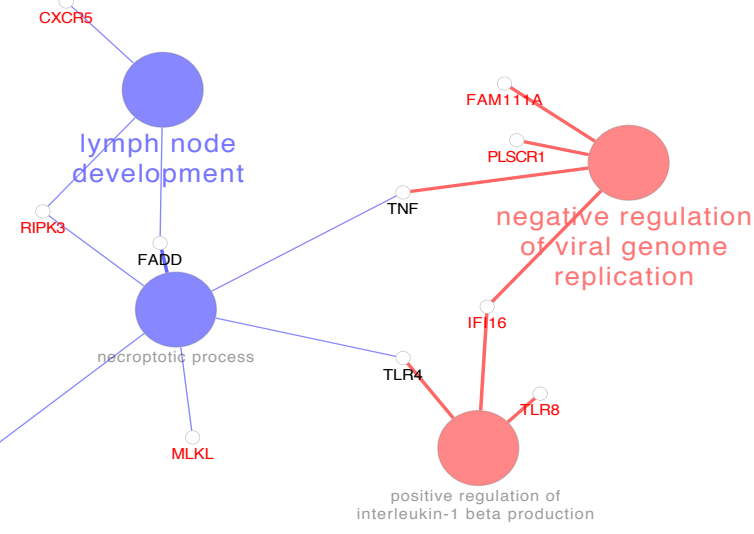


Figure 2
A
$\square$ Group 1P ( $\mathrm{n}=12)$
$\square$ Group 2P ( $n=12)$
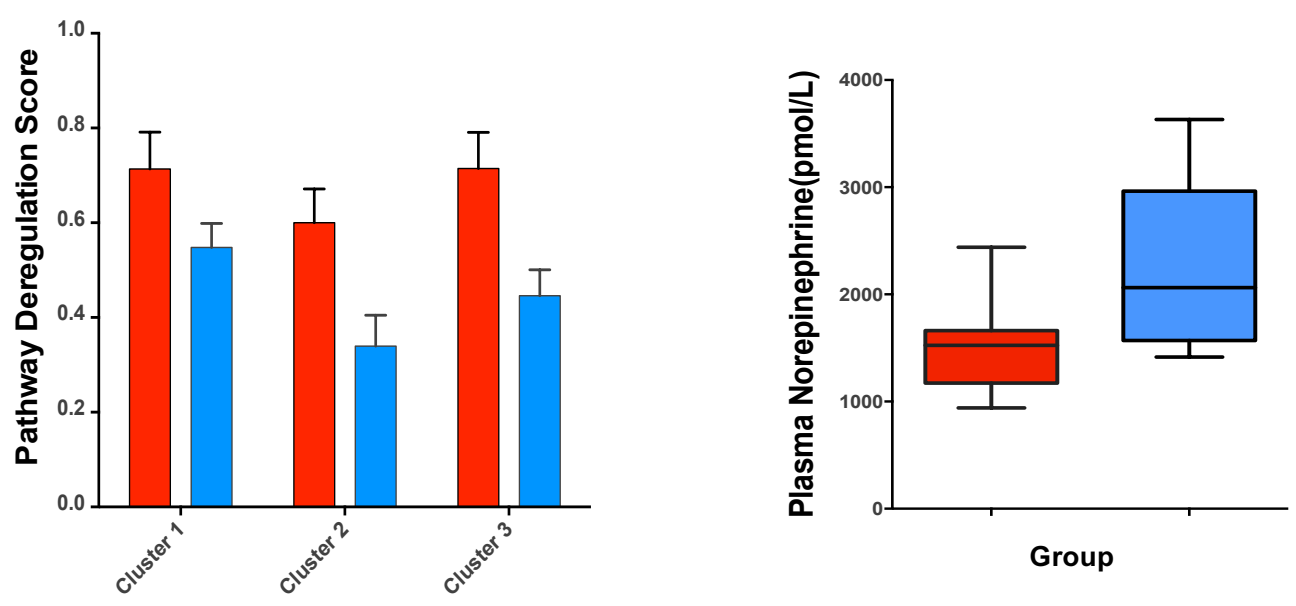

B

E
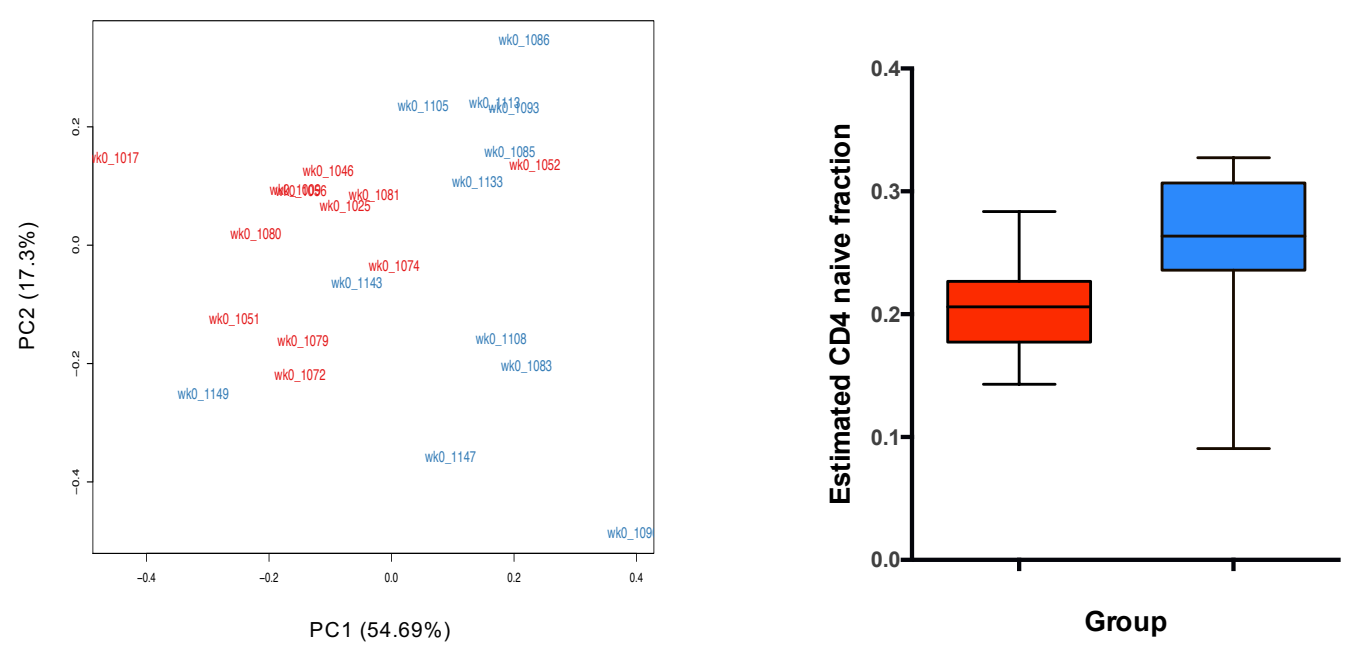

C

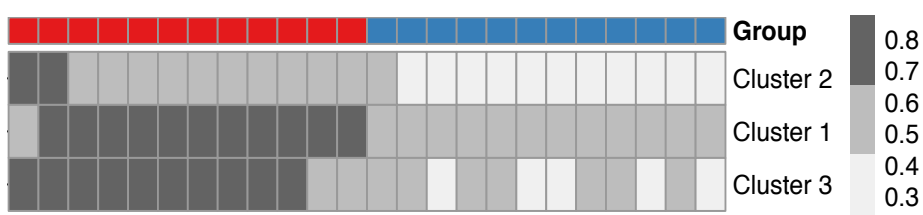


Figure 3

A

CFQ over time

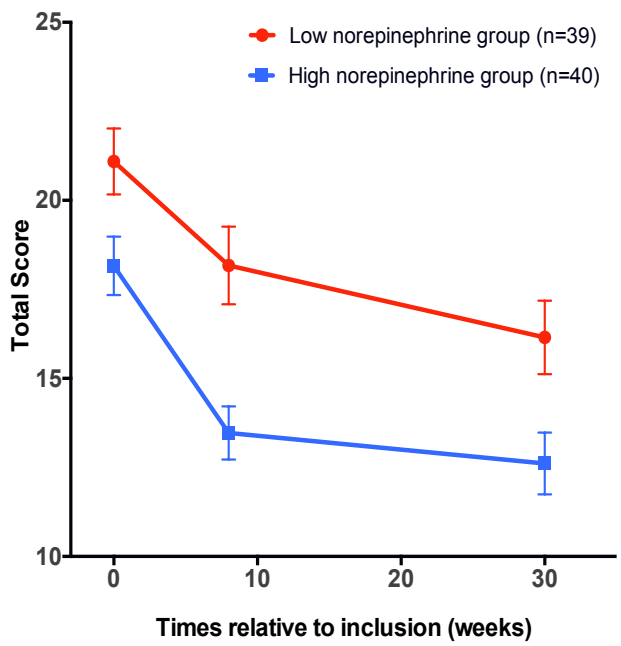

B

\section{FDI over time}

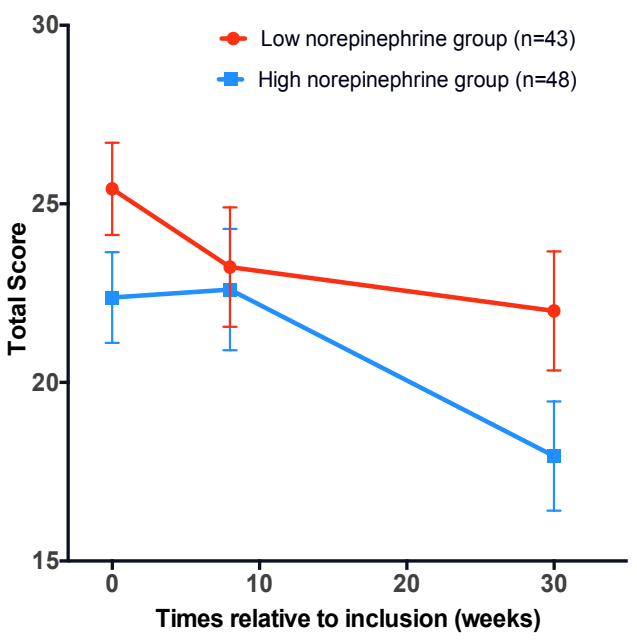


Figure S1

\section{PATHIFIER ANALYSIS}

UNSUPERVISED CLUSTERING

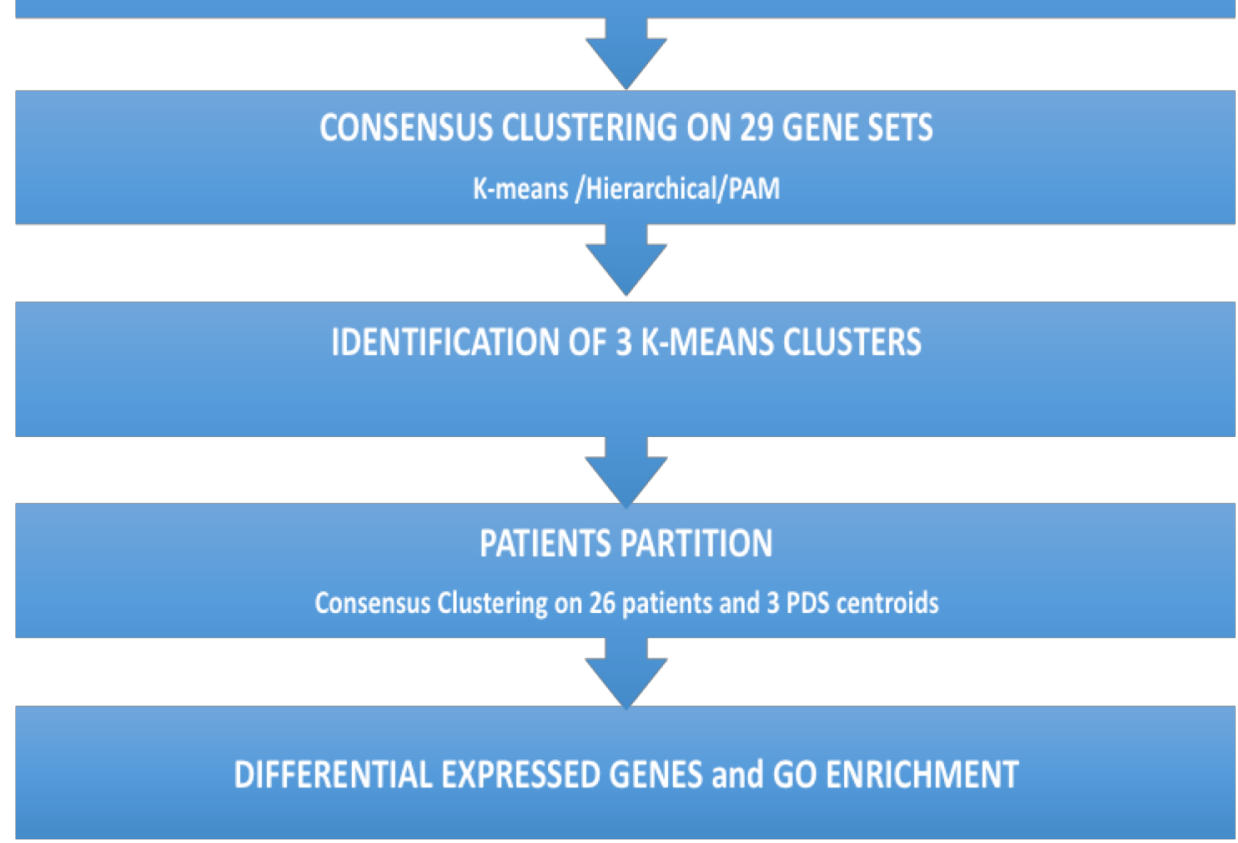

Fig S1. Computational Workflow. Pathifier analysis identified the top 29 deregulated gene sets and unsupervised clustering suggested subgroups of patients. Then supervised method was used to classify gene sets into $3 \mathrm{~K}$ Means clusters of which the centroids were the centers of the clusters. Subsequently, those three centroids were used to partition patients. Differentially expressed genes were matched with gene contents of the C7 MSigDB. GO enrichment by ClueGO was performed. 


\section{Figure S2}

\section{Extreme cases}

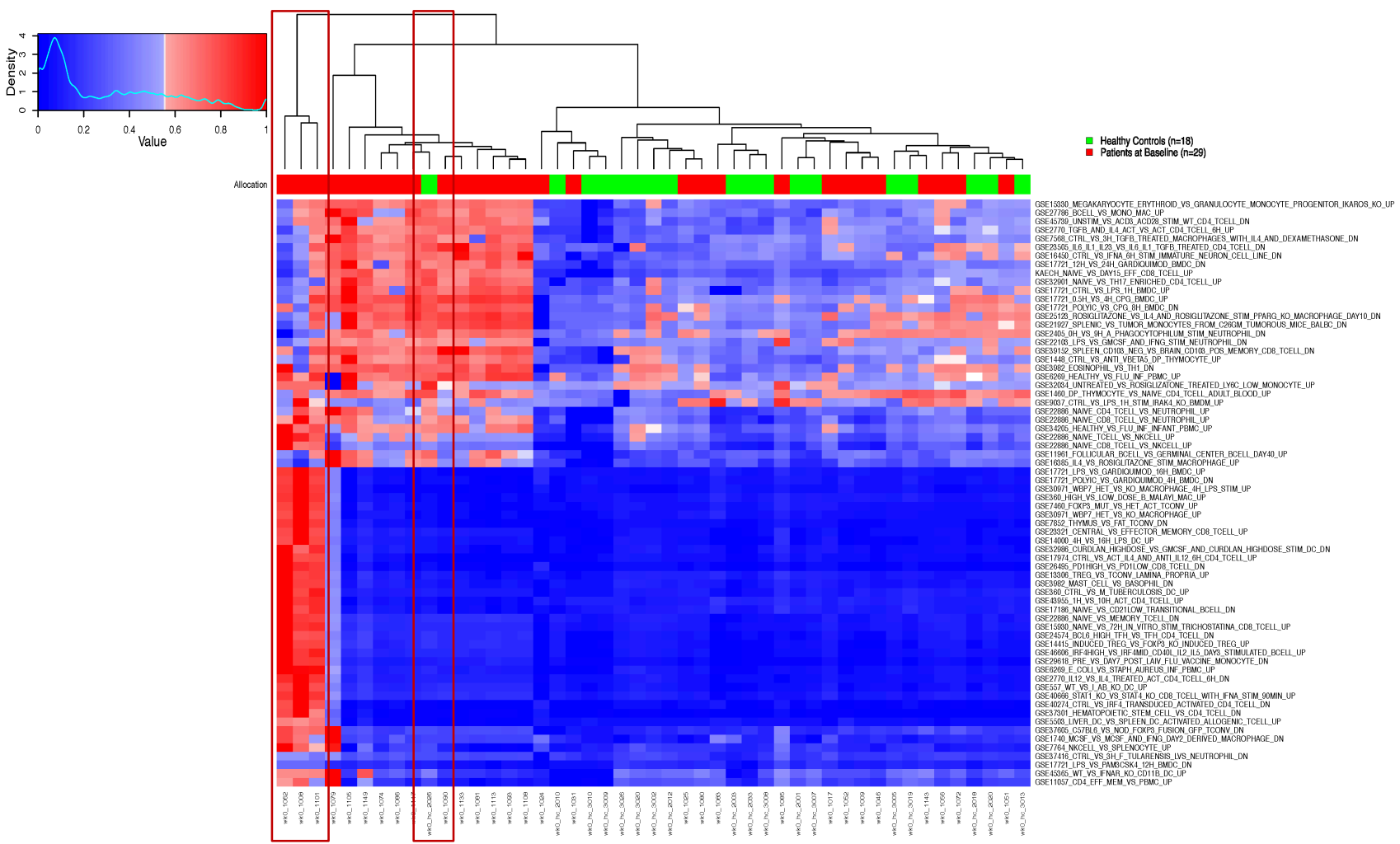

Fig S2. Preliminary analysis. Hierarchical clustering of the top deregulated C7 gene sets allowed detection of outliers/extreme cases as shown in the two red frames (3 patients and 1 controls). 
Figure S3

A. Consensus clustering

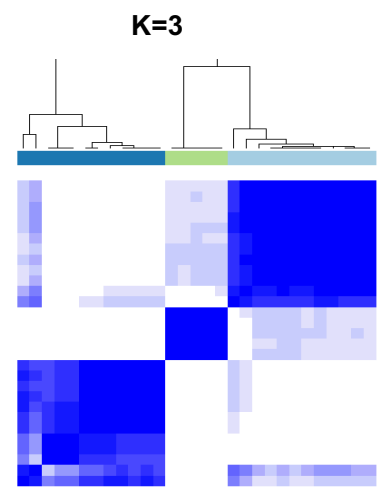

\section{\begin{tabular}{|l|l|}
\hline$ㅁ$ & 1 \\
\hline & 2 \\
\hline & 3 \\
\hline
\end{tabular}}
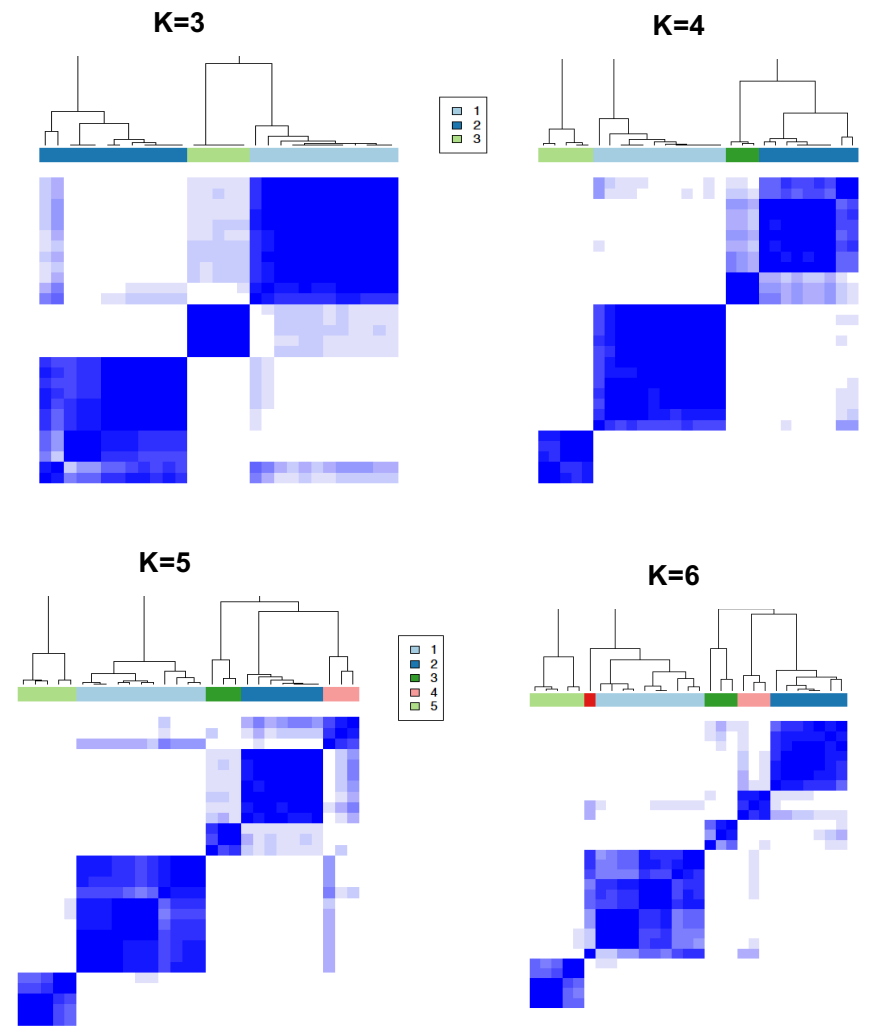

Consensus CDF

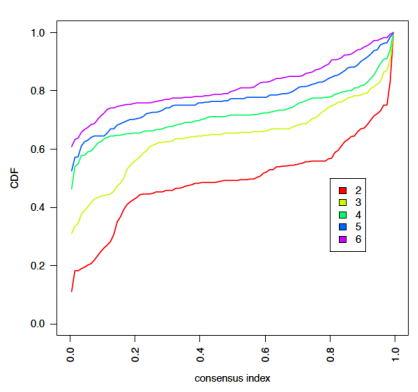

B. Silhouette plot

$k=3$
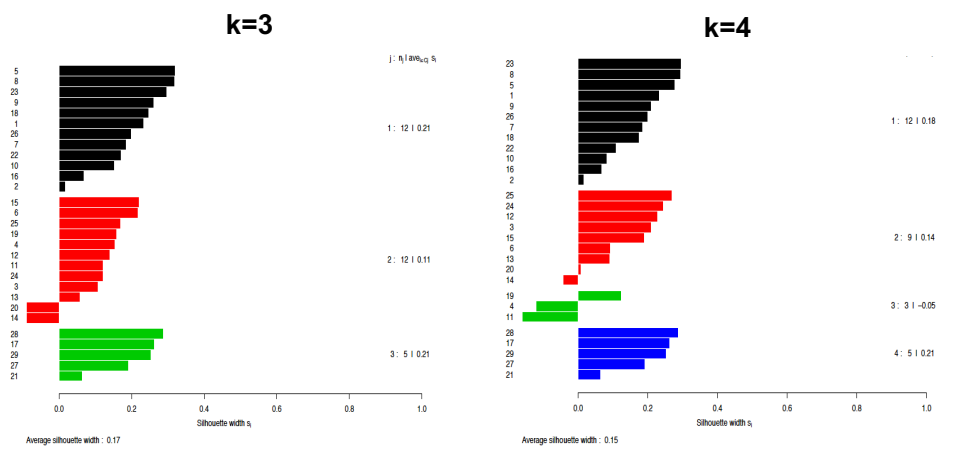

\section{K- Means clusters}

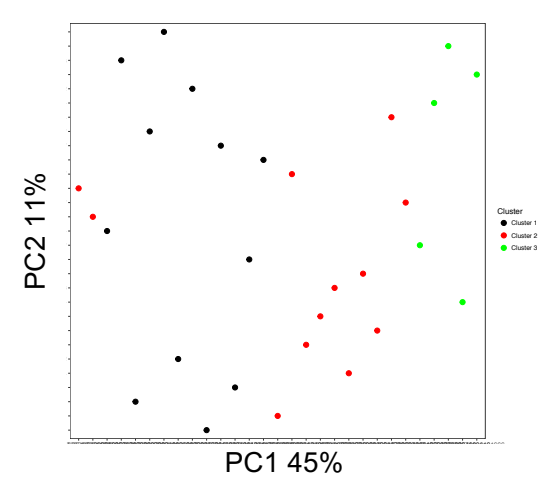


Fig S3. Consensus clustering of the 29 deregulated gene sets

A. Visualization of consensus matrices from $K$-means consensus clustering for $K=3$, 4, 5 and 6 target clusters. Number of $K$-means clusters were derived using consensus clustering based on the 29 gene sets x 43 individuals matrix, exploring the range of 2 to $6 \mathrm{~K}$-means clusters on the 29 gene sets (Only results of $\mathrm{k}$ from 3-6 were shown). Consensus cumulative distribution function $(\mathrm{CDF})$ and delta area (change in $\mathrm{CDF}$ area) plots for 2-6 clusters. The delta plot when $k=3$ showed the optimal change of segregated groups observed.

B. Silhouette plots were generated to evaluate the coherence of the clustering of the 29 gene sets. Silhouette plots when $K=3$ or $K=4$ showed the clearer partitioning for $K=3$.

C. PCA inspecting PDS variances of the 29 gene sets colored by their corresponding K-Means clusters. Black: Cluster 1, Red: Cluster 2, Green: Cluster 3. 
Figure S4

A

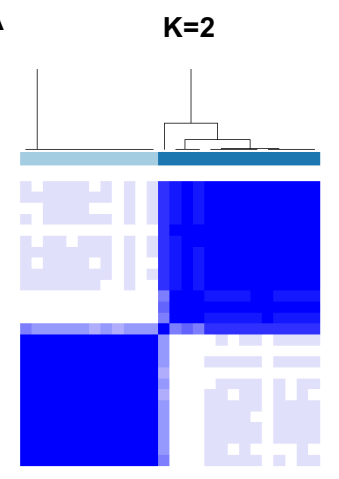

B

Silhouette plot $k=2$

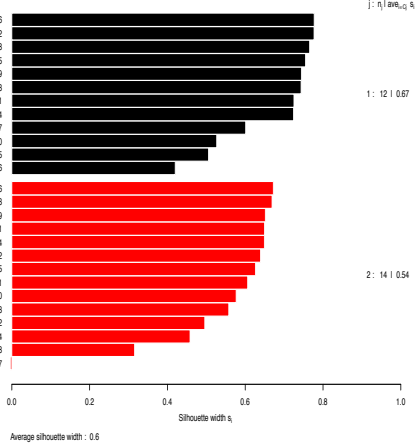

C

Consensus CDF

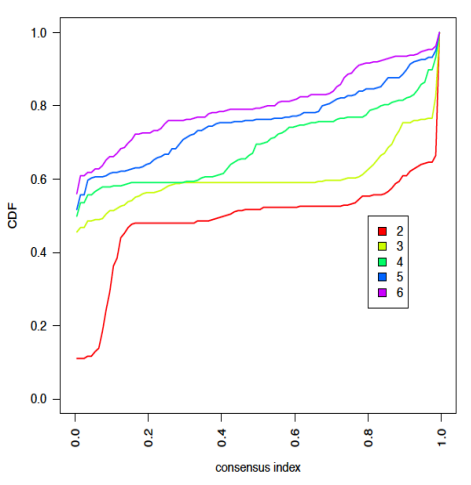

\section{圆}

$K=3$

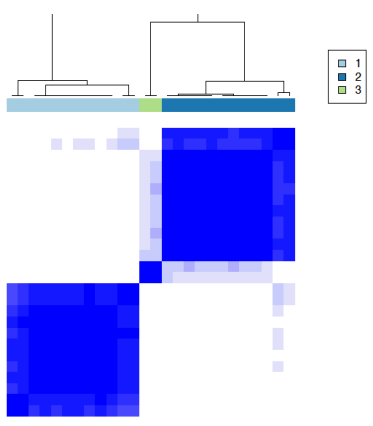

Silhouette plot k=3

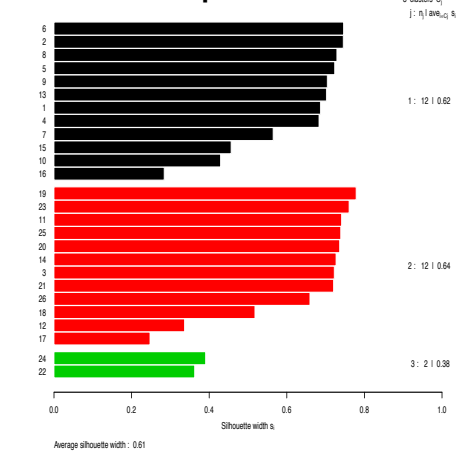

Silhouette plot $k=4$
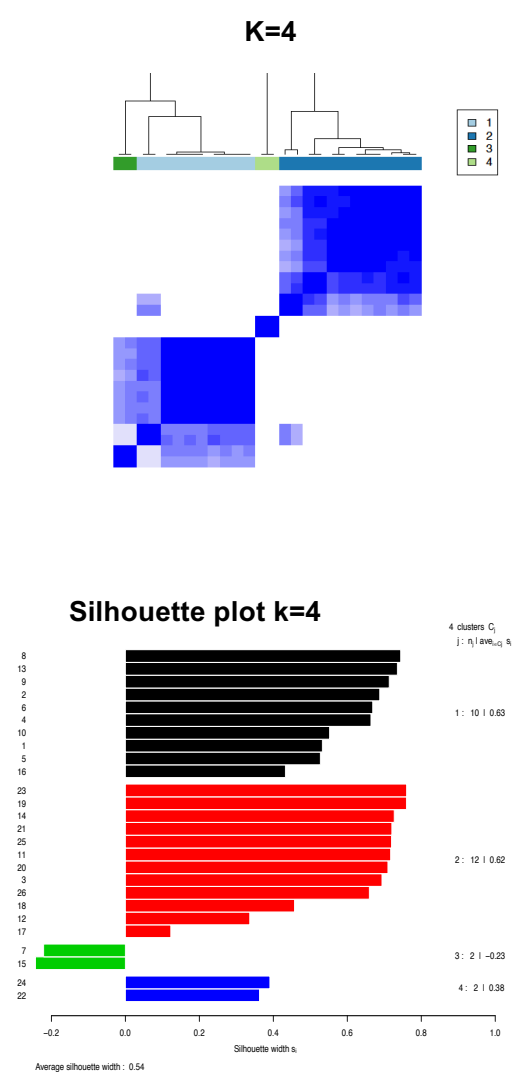

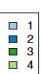

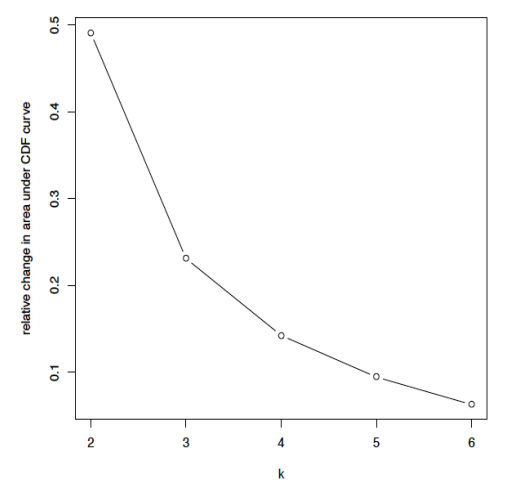


Fig S4. Partitioning of patients using consensus clustering

A. Visualization of consensus matrices from $K$-means consensus clustering. Number of $K$-means clusters were derived using consensus clustering based on 3 centroids of the 26 patients, exploring the range of 2 to $4 \mathrm{~K}$-means clusters.

B. Silhouette plots for $K=3$ clusters showed the clearest partitioning.

C. Consensus CDF and delta area (change in CDF area) plots for 2-4 clusters. The delta plot when $\mathrm{k}=3$ showed the change of robustly segregated groups. 